\title{
NONCOLLINEAR PHASE MATCHED FOUR PHOTON FREQUENCY MIXING IN WATER
}

\author{
A. PENZKOFER, J. KRAUS and J. SPERKA \\ Naturwissenschaftliche Fakultät II - Physik, Universität Regensburg, \\ 8400 Regensburg, Fed. Rep. Germany
}

Received 29 January 1981

\begin{abstract}
An intense ultraviolet picosecond light pulse at $\omega_{S}=2 \gamma_{\mathrm{P}}-\omega_{\mathrm{L}}$ is generated in water by noncollinear phase matched nonresonant four photon frequency mixing of two input picosecond light pulses at frequencies $\tilde{\nu}_{\mathrm{P}}=18960 \mathrm{~cm} \mathrm{cmd}^{-1}$ and $\tilde{\nu}_{\mathrm{L}}=$ $9480 \mathrm{~cm}^{-1}$. An energy conversion of up to $W_{\mathrm{S}} / W_{\mathrm{L}}=0.07$ was achieved. The nonlinear susceptibility components were determined to be $x_{y y y y}^{(3)}\left(-\omega_{S} ; \omega_{P}, \omega_{P},-\omega_{L}\right)=7.5 \times 10^{-34} \mathrm{Cm} / \mathrm{V}^{3}$ and $x_{y x x y}^{(3)}\left(-\omega_{S} ; \omega_{P}, \omega_{P},-\omega_{L}\right)=2.4 \times 10^{-34} \mathrm{Cm} / \mathrm{V}^{3}$.
\end{abstract}

\section{Introduction}

Four photon interactions are caused by the third order nonlinear polarization $\chi^{(3)}$ EEE which is the lowest order nonlinear polarization term in isotropic media $[1-3]$. Four photon frequency mixing processes may be resonantly enhanced when single frequencies, sum frequencies, or difference frequencies approach a transition frequency from initial state to an excited state $[4,5]$. The frequency mixing depends strongly on the phase mismatch between the involved waves [1-5] and optimum conversion efficiency is obtained for perfect phase matching.

In this letter nonresonant, noncollinear phase matched four photon frequency mixing $[6,7]$ of picosecond light pulses in water is studied. A high conversion efficiency is obtained. The nonlinear susceptibility components are determined.

\section{Theory}

Input pulses of frequencies $\omega_{\mathrm{P}}$ and $\omega_{\mathrm{L}}$ generate light at $\omega_{S}=2 \omega_{P}-\omega_{L}$. The wave vector geometry is seen in fig. 1. The phase mismatch is defined as $\Delta k=k_{\mathrm{S}}+k_{\mathrm{L}}-2 k_{\mathrm{P}}$. The electric field $E_{\mathrm{L}}$ is directed parallel to the $y$-axis while the electric field $E_{\mathrm{P}}$ is either parallel to the $x$-axis or to the $y$-axis.

The noncollinear frequency mixing is described by

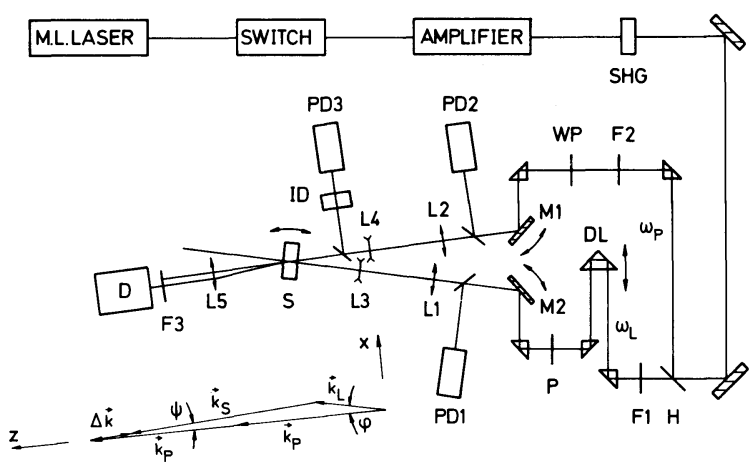

Fig. 1. Experimental setup. SHG, KDP crystal for second harmonic generation; $\mathrm{H}$, harmonic beam splitter; $\mathrm{F} 1-\mathrm{F} 3$, filters; WP, $\lambda / 2$ waveplate; DL, optical delay line; P, polarizer; M1, M2, plane mirrors; PD1-PD3, vacuum photocells, L1, L2 convex cylindrical lenses $(f=30 \mathrm{~cm}) ; \mathrm{L} 3$, L4 concave cylindrical lenses $(f=-10 \mathrm{~cm})$. Composite focal lengths are $f(\mathrm{~L} 1-\mathrm{L} 3)=35 \mathrm{~cm}$ and $f(\mathrm{~L} 2-\mathrm{L} 4)=60 \mathrm{~cm}$. ID, two photon absorber $\mathrm{CH}_{2} \mathrm{I}_{2}(1 \mathrm{~cm})$ for intensity detection; $\mathrm{S}$, water sample; L5 collimating lens $(f=10 \mathrm{~cm})$; D, optical multichannel analyser or photodetector.

the wave equation [5]

$$
\begin{aligned}
& \nabla^{2} E-\frac{n^{2}}{c^{2}} \frac{\partial^{2}}{\partial t^{2}} \boldsymbol{E}-\frac{\alpha n}{c} \frac{\partial}{\partial t} \boldsymbol{E} \\
&=\mu_{0}\left[\frac{\partial^{2}}{\partial t^{2}} \boldsymbol{P}_{\mathrm{NL}}-\frac{c^{2}}{n^{2}} \nabla\left(\nabla \cdot \boldsymbol{P}_{\mathrm{NL}}\right)\right],
\end{aligned}
$$


$n$ is the refractive index and $\alpha$ is the absorption coefficient. Eq. (1) is solved with the ansatz

$A=\frac{1}{2} \sum_{\gamma=\mathrm{P}, \mathrm{L}, \mathrm{S}} A_{\gamma} \exp \left[\mathrm{i}\left(k_{\gamma} r-\omega_{\gamma} t\right)\right]+$ c.c.,

where $\boldsymbol{A}$ stands for $\boldsymbol{E}$ and $\boldsymbol{P}_{\mathrm{NL}}$. Using the slowly varying envelope approximation and the substitutions $z^{\prime}=$ $z, y^{\prime}=y-\left(k_{\gamma y} / k_{\gamma z}\right) z, x^{\prime}=x-\left(k_{\gamma x} / k_{\gamma z}\right) z, t^{\prime}=$ $t-\left(\omega_{\gamma} n_{\mathrm{g} \gamma} / k_{\gamma z} c^{2}\right) z$ one obtains

$$
\begin{aligned}
& \frac{\partial}{\partial z^{\prime}} \boldsymbol{E}_{\gamma}+\frac{n_{\mathrm{g} \gamma} \alpha_{\gamma} \omega_{\gamma}}{2 k_{\gamma z} c} \boldsymbol{E}_{\gamma} \\
& \quad=\frac{\mathrm{i}}{2 k_{\gamma z}} \mu_{0}\left[\omega_{\gamma}^{2} \boldsymbol{P}_{\mathrm{NL}, \gamma}-\frac{c^{2}}{n_{\gamma}^{2}} \boldsymbol{k}_{\gamma}\left(\boldsymbol{k}_{\gamma} \cdot \boldsymbol{P}_{\mathrm{NL}, \gamma}\right)\right],
\end{aligned}
$$

$n_{\mathrm{g} \gamma}=n_{\nu} /\left[1+\left(\lambda_{\gamma} / n_{\gamma}\right) \partial n_{\gamma} / \partial \lambda_{\gamma}\right]$ is the group refractive index at frequency $\omega_{\gamma}$. Depletion of the input pulses is neglected in the mixing process and eq. (3) is only solved for $\boldsymbol{E}_{\mathrm{S}}$.

The nonlinear polarization is approximated by the lowest order nonlinear term of isotropic media $\boldsymbol{P}_{\mathrm{NL}}=$ $\chi^{(3)}: \boldsymbol{E E E}$. The symmetry properties of $\chi^{(3)}[2,5]$ together with the polarization of the input laser pulses $\left(E_{\mathrm{L}} \| y\right.$-axis, $E_{\mathrm{P}} \| y$ or $x$-axis) lead to

$$
\begin{aligned}
& P_{\mathrm{NL}, \mathrm{S}, y}=\frac{3}{4} \chi_{y j j y}^{(3)}\left(-\omega_{\mathrm{S}} ; \omega_{\mathrm{P}}, \omega_{\mathrm{P}},-\omega_{\mathrm{L}}\right) \\
& \quad \times E_{\mathrm{P}}^{2} E_{\mathrm{L}}^{*} \exp (-\mathrm{i} \Delta k r)
\end{aligned}
$$

and

$$
\begin{aligned}
& P_{\mathrm{NL}, \mathrm{S}, x}=P_{\mathrm{NL}, \mathrm{S}, z}=0 \\
& \left(j=x \text { for } E_{\mathrm{P}} \| x, j=y \text { for } E_{\mathrm{P}} \| y, \boldsymbol{k}_{\mathrm{S}} \perp \boldsymbol{P}_{\mathrm{NL}, \mathrm{S}}\right) . \\
& \text { Integration of eq. (3) with the initial condition } \\
& E_{\mathrm{S}}(z=0)=0 \text { gives } \\
& E_{\mathrm{S}}\left(x^{\prime}, y^{\prime}, t^{\prime}, l\right) \\
& \quad=\mathrm{i} \frac{3 \mu_{0} \omega_{\mathrm{S}} c}{8 n_{\mathrm{S}} \cos (\psi)} \chi_{y j j y}^{(3)} \exp \left(-\mathrm{i} \Delta k_{x} x^{\prime}\right) \\
& \quad \times \int_{0}^{l} E_{\mathrm{P}}^{2}\left(x^{\prime}, y^{\prime}, t^{\prime}, z\right) E_{\mathrm{L}}^{*}\left(x^{\prime}, y^{\prime}, t^{\prime}, z\right) \\
& \quad \times \exp \left\{-\mathrm{i}\left[\Delta k_{z}-\Delta k_{x} \tan (\psi)\right] z-\frac{n_{\mathrm{gS}} \alpha_{\mathrm{S}}(l-z)}{2 n_{\mathrm{S}} \cos (\psi)}\right\} \mathrm{d} z,
\end{aligned}
$$

where $k_{\mathrm{S} z}=n_{\mathrm{S}} \omega_{\mathrm{S}} \cos (\psi) / c$ and $x=x^{\prime}-\tan (\psi) z$ are used $\left(E_{\mathrm{S} y}=E_{\mathrm{S}}, E_{\mathrm{S} x}=E_{\mathrm{S} z}=0\right)$. The phase mismatch is $\Delta k_{x}=k_{\mathrm{L}} \sin (\varphi)-k_{\mathrm{S}} \sin (\psi)$ and $\Delta k_{z}=k_{\mathrm{L}} \cos (\varphi)$ $+k_{\mathrm{S}} \cos (\varphi)-2 k_{\mathrm{P}}$. The pump pulse amplitudes are

$$
\begin{aligned}
& E_{\mathrm{P}}(x, y, z, t)=E_{0 \mathrm{P}} \exp \left\{-\frac{1}{2}\left[x^{2} / x_{\mathrm{P}}^{2}+y^{2} / y_{\mathrm{P}}^{2}\right.\right. \\
& \left.\left.\quad+\left(t-n_{\mathrm{gP}^{2}} z / c\right)^{2} / t_{\mathrm{P}}^{2}+\alpha_{\mathrm{P}} z\right]\right\},
\end{aligned}
$$

and

$$
\begin{aligned}
& E_{\mathrm{L}}(x, y, z, t) \\
& \quad=E_{0 \mathrm{~L}} \exp \left(-\frac{1}{2}\left\{[x \cos (\varphi)-(z-l / 2) \sin (\varphi)]^{2} / x_{\mathrm{L}}^{2}\right.\right. \\
& \quad+y^{2} / y_{\mathrm{L}}^{2}+\left[t-t_{\mathrm{D}}-n_{\mathrm{gL}}(x \sin (\varphi)+z \cos (\varphi)) / c\right]^{2} / t_{\mathrm{L}}^{2} \\
& \left.\left.\quad+\alpha_{\mathrm{L}}[x \sin (\varphi)+z \cos (\varphi)]\right\}\right) .
\end{aligned}
$$

In eq. (7) a coordinate transformation of $\boldsymbol{E}_{\mathrm{L}}$ to the $x, y, z$-frame of $E_{\mathrm{P}}$ is performed. The pump beams cross in the center of the cell due to term $(l / 2) \sin (\varphi)$ in eq. (7). $t_{\mathrm{D}}$ represents the temporal separation of the input pulses.

The intensity of the generated light signal is obtained from eq. (5) by averaging $E_{\mathrm{S}}$ over fast changes in the $x^{\prime}$ direction [15] and by forming the absolute square $I_{\mathrm{S}}=n_{\mathrm{S}} c \epsilon_{0}\left|E_{\mathrm{S}}\right|^{2} / 2$. The generated signal energy is calculated by integration over space and time

$W_{\mathrm{S}}=\int_{-\infty}^{\infty} \int_{-\infty}^{\infty} \int_{-\infty}^{\infty} I_{\mathrm{S}} \mathrm{d} x^{\prime} \mathrm{d} y^{\prime} \mathrm{d} t^{\prime}$

and finally, the energy conversion $\eta=W_{\mathrm{S}} / W_{\mathrm{L}}$ is defined as the ratio of generated energy at $\omega_{S}$ to input energy at $\omega_{\mathrm{L}}$. The experimental parameters involved in the calculations are summarized in table 1 .

\section{Experiments}

The experimental setup is depicted in fig. 1. A mode locked Nd-phosphate glass laser is used [8]. Single light pulses are selected with an electrooptical switch and increased in energy with an amplifier. The pulses have a duration of $\Delta t_{\mathrm{L}} \approx 6 \mathrm{ps}$ (FWHM) and a spectral width of $\Delta \widetilde{\nu}_{\mathrm{L}} \approx 10 \mathrm{~cm}^{-1}$ (FWHM). The second harmonic is generated in a KDP crystal (length $1 \mathrm{~cm})$. The fundamental laser pulse $\left({\widetilde{v_{L}}}_{\mathrm{L}} 9480 \mathrm{~cm}^{-1}\right)$ and the second harmonic $\left(\widetilde{\nu_{P}}=18960 \mathrm{~cm}^{-1}\right)$ act as 
Table 1

Laser and material parameters

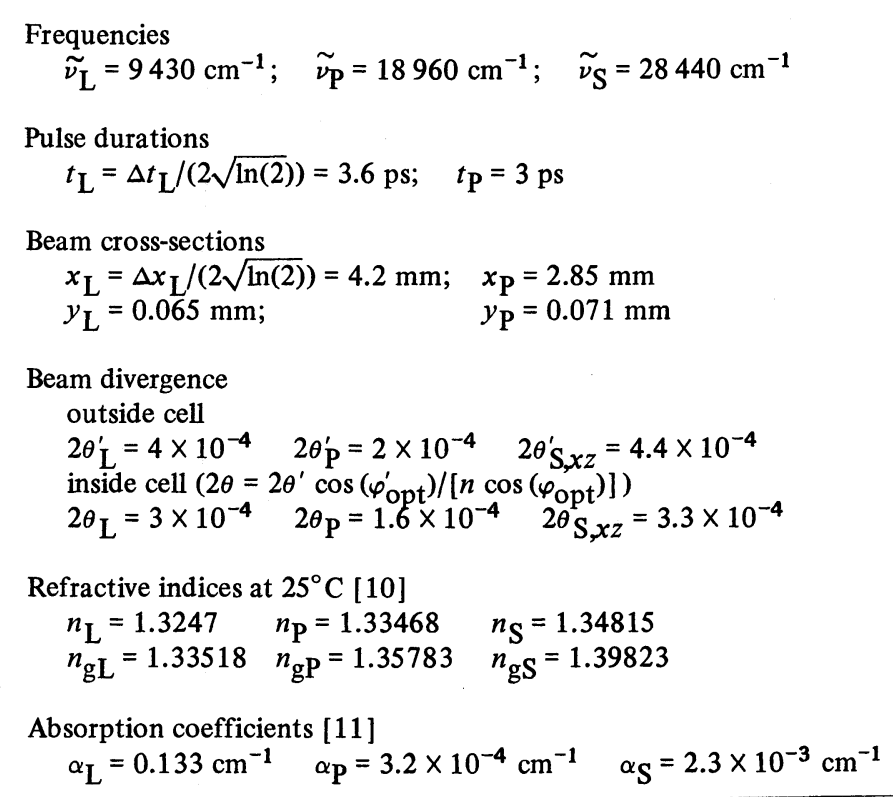

input pulses in the frequency mixing process.

The picosecond pulses at frequencies $\omega_{P}$ and $\omega_{L}$ are separated with a harmonic beam splitter $H$. The optical delay line DL adjusts the pulses for temporal overlap in the sample. The angle $\varphi^{\prime}$ between the input beams (outside sample) is changed by translation and rotation of the mirrors $\mathrm{M} 1$ and $\mathrm{M} 2$. The composite cylindrical lenses L1, L3 and L2, L4 form line focuses in the sample $\mathrm{S}$. The beam extension in $x$-direction assures spatial overlap of the waves over a long distance (ca. $3 \mathrm{~cm}$ ). Fine tuning of the angle $\varphi$ (inside cell) is achieved by twisting the sample. The change of refraction angle between input beams is approximately $0.01^{\circ}$ for cell rotation of $1^{\circ}$.

The peak intensity of the input pulses at frequency $\omega_{\mathrm{P}}$ is monitored by two-photon transmission measurements through methyliodide ID with photo-cells PD2 and PD3 [9]. The pulse energy at frequency $\omega_{L}$ is measured with the calibrated photodetector PD1. The generated light at frequency $\widetilde{\nu}_{\mathrm{S}}=28440 \mathrm{~cm}^{-1}$ is detected either with an optical multichannel analyser (energy and space resolution) or with a calibrated photodetector.

A $\lambda / 2$ wave plate WP is used to orient $E_{\mathrm{P}}$ parallel to $E_{\mathrm{L}}$ (coupling constant $\chi_{\text {yyyy }}^{(3)}$ ). Without the waveplate,
$\boldsymbol{E}_{\mathrm{P}}$ is directed in the $x$-direction (coupling constant $\left.\chi_{y x x y}^{(3)}\right)$. Polarizer $\mathrm{P}$ establishes perfect polarization of $E_{\mathrm{L}}$ in $y$-direction.

\section{Results}

The angular dependence of the energy conversion $\eta=X_{\mathrm{S}} W_{\mathrm{L}}$ is depicted in fig. 2 . The dashed curve $\eta(\varphi) / \eta\left(\varphi_{\text {opt }}\right)$ is calculated with the aid of eq. (5). The solid curve is obtained by convolution of the dashed curve with the angular intensity distribution of the input lasers. The optimum energy conversion is reduced by the divergence of the input beams by a factor of 0.65 . The experimental points $(O)$ are adjusted to the calculated curves at the theoretical phase matching angle $\varphi_{\mathrm{opt}}=\operatorname{arc} \cos \left\{\left[\left(2 k_{\mathrm{P}}\right)^{2}+k_{\mathrm{L}}^{2}-k_{\mathrm{S}}^{2}\right] /\right.$ $\left(4 k_{\mathrm{P}} k_{\mathrm{L}}\right)=10.685^{\circ}(\Delta k=0)$. Experimentally, an angle of $\varphi_{\text {opt }}=10.63^{\circ} \pm 0.08^{\circ}\left(\varphi_{\text {opt }}^{\prime}=14.15^{\circ} \pm 0.1^{\circ}\right.$ outside water) was found. The angular spread of the phase matching angle is $\Delta \varphi_{\mathrm{opt}}=0.024^{\circ}=4.2 \times 10^{-4}$ $\operatorname{rad}$ (FWHM).

The angle $\psi$ between $k_{\mathrm{P}}$ and $k_{\mathrm{S}}$ is given by the wavevector triangle included in fig. 1. (Deviation from a triangular geometry increases $|\Delta k|)$. One finds $\psi=$ arc 


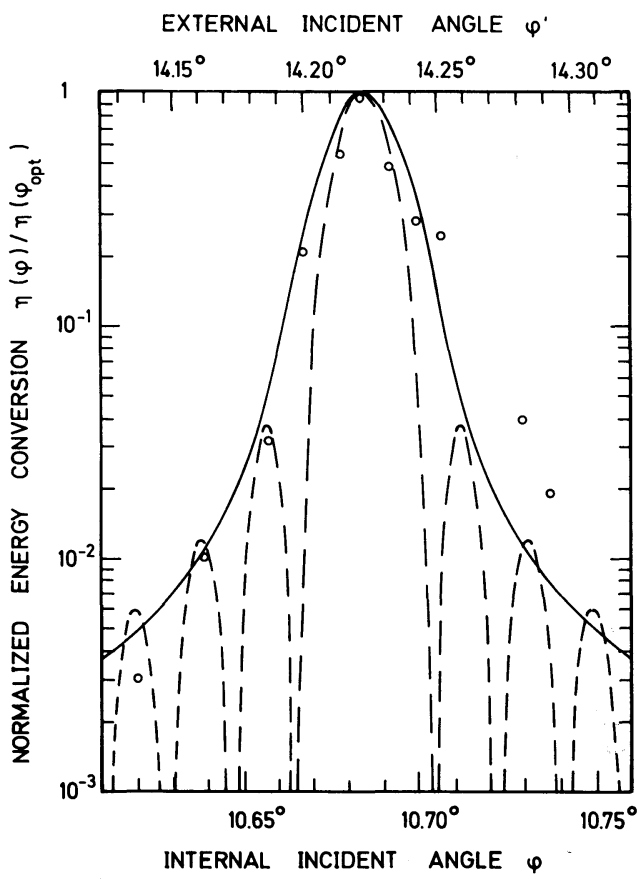

Fig. 2. Angular dependence of energy conversion. Dashed curve, calculated with aid of eq. (5). Solid curve, convolution of dashed curve with angular intensity distribution of input pulses (see table 1). The data points are adjusted to position of peak conversion. External incident angle $\varphi^{\prime}$ is calculated for cell orientation normal to bisector of input beams $(\varphi=\arcsin$ $\left.\left[\left(1 / n_{\mathrm{L}}\right) \sin \left(\varphi^{\prime} / 2\right)\right]+\arcsin \left[\left(1 / n_{P}\right) \sin \left(\varphi^{\prime} / 2\right)\right]\right)$.

$\sin \left\{k_{\mathrm{L}} \sin (\varphi) /\left[k_{\mathrm{L}}^{2}+\left(2 k_{\mathrm{P}}\right)^{2}-4 k_{\mathrm{P}} k_{\mathrm{L}} \cos (\varphi)\right]^{1 / 2}\right\}$. For perfect phase matching the angle of emission is $\psi_{\mathrm{opt}}$ $=\arcsin \left[k_{\mathrm{L}} \sin (\varphi) / k_{\mathrm{S}}\right]=3.48^{\circ}\left(\psi_{\text {opt }}^{\prime} \approx 4.85^{\circ}\right.$ outside sample).

The divergence of the generated light in the $x z$ plane was measured with an optical multichannel analyser positioned in the focal plane of lens L5 (see fig. 1). A divergence $2 \theta_{\mathrm{S}, x z}^{\prime}=4.4 \times 10^{-4} \mathrm{rad}$ was found.

At large phase mismatch angles $\left|\varphi-\varphi_{\text {opt }}\right|>0.5^{\circ}$ light of frequency $\omega_{\mathrm{S}}$ is observed at three emission angles. The strongest beam is radiated in the above described direction. The two other beams are due to phase matched interaction of one input pulse with stray light of the other input pulse [12].

The dependence of the energy conversion on the input peak intensity $I_{0 \mathrm{P}}$ is shown in fig. 3 for various sample lengths. The calculated curves and measured data points are presented for perfect phase matching.

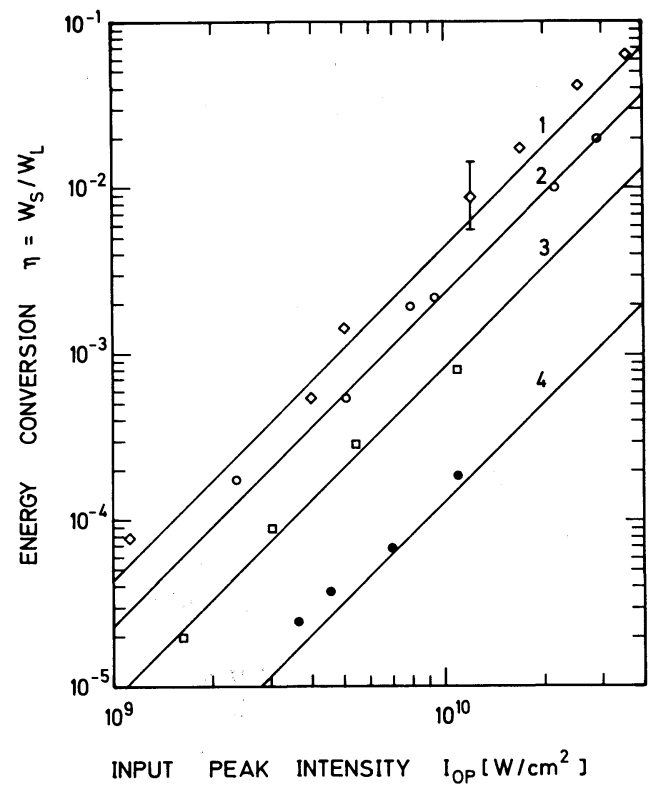

Fig. 3. Energy conversion versus input peak intensity $I_{0} \mathrm{P}$ for various sample lengths at perfect noncollinear phase-matching. $E_{\mathrm{P}}\left\|E_{\mathrm{L}}\right\| y$-axis. Sample lengths $l=2 \mathrm{~cm}(1, \diamond), l=1 \mathrm{~cm}(2, \circ)$, $l=0.5 \mathrm{~cm}(3, \square)$ and $l=0.2 \mathrm{~cm}(4, \bullet)$. Curves are calculated for $x_{y y y y}^{(3)}=7.5 \times 10^{-34} \mathrm{Cm} / \mathrm{V}^{3}$.

At a fixed cell length the energy conversion increases quadratically with $I_{0 \mathrm{P}}$. At $I_{0 \mathrm{P}}=4 \times 10^{10} \mathrm{~W} / \mathrm{cm}^{2}$ an energy conversion of $\eta \approx 0.07$ was obtained in a water cell of $2 \mathrm{~cm}$ length $\left(I_{0 \mathrm{~L}} \approx 5 \times 10^{8} \mathrm{~W} / \mathrm{cm}^{2}\right)$. For $I_{0 \mathrm{P}}>2 \times 10^{10} \mathrm{~W} / \mathrm{cm}^{2}$ the four photon interaction is more complex than described by eq. (5): i) The frequency mixing process $2 \omega_{\mathrm{P}}-\omega_{\mathrm{L}} \rightarrow \omega_{\mathrm{S}}$ changes over to the parametric interaction process $\omega_{\mathrm{P}}+\omega_{\mathrm{P}} \rightarrow$ $\omega_{L}+\omega_{S}$ where light at frequencies $\omega_{L}$ and $\omega_{S}$ is amplified (complete equation system 3 should be solved) $[13,14]$. ii) When $I_{0 \mathrm{~L}} \geqslant I_{0 \mathrm{P}}$ depletion of the pump pulse at $\omega_{\mathrm{P}}$ has to be taken into account. At $I_{0 \mathrm{P}}=$ $3 \times 10^{10} \mathrm{~W} / \mathrm{cm}^{2}$ the energy conversion reduced from $\eta=0.05$ for $I_{0 \mathrm{~L}} \approx 5 \times 10^{8} \mathrm{~W} / \mathrm{cm}^{2}$ to 0.035 for $I_{0 \mathrm{~L}} \approx$ $3 \times 10^{10} \mathrm{~W} / \mathrm{cm}^{2}$. For $I_{0 \mathrm{~L}}>5 \times 10^{10} \mathrm{~W} / \mathrm{cm}^{2}$ light at frequency $\omega_{L}$ is reduced by generation of broad band picosecond light continua due to parametric four photon interaction $[13,16]$.

At a fixed input intensity $I_{0 \mathrm{P}}$ the energy conversion increases quadratically with sample length up to a length of $1 \mathrm{~cm}$. For longer cells the conversion levels down since the spatial overlap of the noncollinear input beams is limited to about $3 \mathrm{~cm}$. For cell lengths 
longer than $3 \mathrm{~cm}$ and optimum overlap in the center of the cell the conversion $\eta$ reduces gradually since the input pulse at $\omega_{\mathrm{L}}$ is slightly absorbed in water.

The duration of the generated light at $\omega_{\mathrm{S}}$ is determined by the temporal overlap of the input pulses. Calculations of $I_{\mathrm{S}}(t)$ indicate a pulse duration of $\Delta t_{\mathrm{S}}$ $\approx 3.5 \mathrm{ps}$ (FWHM).

The spectral width of the generated light was measured with a $0.6 \mathrm{~m}$ spectrograph and an optical multichannel analyser. A spectral width of $\Delta \tilde{\nu}_{\mathrm{S}} \approx 10 \mathrm{~cm}^{-1}$ was found.

The polarization of the output light at $\omega_{\mathrm{S}}$ was analyzed with a polarizer behind the sample. In case of $E_{\mathrm{P}}\left\|E_{\mathrm{L}}\right\| y$ a depolarization ratio of $E_{\mathrm{S}, x}^{2} / E_{\mathrm{S}, y}^{2} \leqslant 0.075$ was measured (coupling constant $\chi_{y x y y}^{(3)}$ ). For $E_{\mathrm{P}} \| x$ and $E_{\mathrm{L}} \| y$ the depolarization was $E_{\mathrm{S}, x}^{2} / E_{\mathrm{S}, y}^{2} \leqslant 0.12$ (coupling constant $\chi_{y x x y}^{(3)}$ ). Theory predicts $E_{\mathrm{S}, x}^{2} / E_{\mathrm{S}, y}^{2}$ $=0\left(E_{S} \| y\right)$ in both cases (see eq. (4)).

The quantitative analysis of the frequency mixing process allows the determination of the nonlinear susceptibility tensor $\chi^{(3)}$. In isotropic media the $\chi^{(3)}$. tensor has three independent components $\chi_{y y y y}^{(3)}$, $\chi_{y x x y}^{(3)}$ and $\chi_{y x y x}^{(3)}[2,5]$. In our case two frequencies are equal and only two components are independent $\left[\chi_{y x y x}^{(3)}=\chi_{y y x x}^{(3)}=\left(\chi_{y y y y}^{(3)}-\chi_{y x x y}^{(3)}\right) / 2\right]$. The two components $\chi_{y y y y}^{3}$ and $\chi_{y x x y}^{3}$ are determined by comparing the measured energy conversion with calculation. We found $\left|\chi_{y y y y}^{(3)}\left(-\omega_{\mathrm{S}} ; \omega_{\mathrm{P}}, \omega_{\mathrm{P}},-\omega_{\mathrm{L}}\right)\right|=(7.5 \pm 2)$ $\times 10^{-34} \mathrm{Cm} / \mathrm{V}^{3}=(6 \pm 1.5) \times 10^{-15}$ esu $\left(\boldsymbol{E}_{\mathrm{P}}\left\|\boldsymbol{E}_{\mathrm{L}}\right\| y\right)$ and $\left|\chi_{y x x y}^{(3)}\left(-\omega_{\mathrm{S}} ; \omega_{\mathrm{P}}, \omega_{\mathrm{P}},-\omega_{\mathrm{L}}\right)\right|=(2.4 \pm 0.6)$ $\times 10^{-34} \mathrm{Cm} / \mathrm{V}^{3}=(1.9 \pm 0.5) \times 10^{-15}$ esu $\left(E_{\mathrm{P}} \| x\right.$, $\left.E_{\mathrm{L}} \| \boldsymbol{y}\right)$. The obtained $\chi^{(3)}$-values are in satisfactory agreement with previously reported nonresonant susceptibility values of water at different frequencies [17-19].

\section{Acknowledgement}

The authors are indebted to Dr. J. Wiedmann for initial measurements and to Prof. M. Maier for helpful discussions. They are grateful to the Deutsche Forschungsgemeinschaft for financial support.

\section{References}

[1] J.A. Armstrong, N. Bloembergen, J. Ducuing and P.S. Pershan, Phys. Rev. 127 (1962) 1918.

[2] P.N. Butcher, Nonlinear optical phenomena (Ohio State University, Ohio, 1965).

[3] N. Bloembergen, Nonlinear optics (Benjamin, New York, 1965).

[4] R.W. Terhune and P.D. Maker, Lasers, Vol. 2, Ch. 4, ed. A.K. Levine (Marcel Dekker, New York, 1968).

[5] C. Flytzanis, Quantum electronics, Vol. 1, Ch. 2, eds. H. Rabin and C.L. Tang (Academic Press, New York, 1975).

[6] D.H. Auston, Appl. Phys. Letters 18 (1971) 250.

[7] D.H. Auston, Optics Comm. 3 (1971) 272.

[8] A. Laubereau and W. Kaiser, Opto-Electronics 6 (1974) 1.

[9] A. Penzkofer and W. Falkenstein, Optics Comm. 17 (1976) 1.

[10] A.M. Hellwege, Landolt-Börnstein, 6th Edition, Vol. 2, Part 8, eds. K.H. Hellwege and A.M. Hellwege (SpringerVerlag, Berlin, 1962).

[11] G.M. Hale and M.R. Querry, Appl. Optics 12 (1973) 555

[12] J.A. Giordmaine, Phys. Rev. Letters 8 (1962) 19.

[13] A. Penzkofer and W. Kaiser, Opt. Quant. Electron 9 (1977) 315 .

[14] A. Penzkofer, A. Laubereau and W. Kaiser, Prog. Quant. Electr. 6 (1979) 55.

[15] G.D. Boyd, A. Ashkin, J.M. Dziedzic and D.A. Kleinman, Phys. Rev. 137 (1965) A1305.

[16] A. Penzkofer, A. Seilmeier and W. Kaiser, Optics Comm. 14 (1975) 363.

[17] P.D. Maker and R.W. Terhune, Phys. Rev. 137 (1965) 801.

[18] D.L. Weinberg, Appl. Phys. Letters 14 (1969) 32.

[19] M. Becker, R. Fischer, J. Frahm, R. Güther and H. Steudel, Opt. Quant. Electron 6 (1976) 279. 